Клады каменных изделий в палеолите - неолите Дальнего Востока: терминологические и функциональные аспекты дискуссии

В рамках данной работы автор обращается к кладам каменных изделий, зафиксированных на территории Дальнего Востока (российская часть, Японский архипелаг) для финала палеолита - неолита/дзёмона в диапазоне 35-2,5 кал. тыс. л.н., и к таким важным аспектам сопровождающей их дискуссии как терминология и функииональное назначение. Для отечественной территории кладов каменных изделий насчитывается всего не более десятка (новопетровская культура на Среднем Амуре, осиповская на Нижнем Амуре, устиновские комплексы в Приморье и ушковские на Камчатке), тогда как для финального палеолита - дзёмона на Японском архипелаге - более 400. Клады существенно различаются по количеству артефактов, их типу, характеру обработки, локализации и сырью. Тем не менее, для обозначения этой категории находок используется практически один термин - как в отечественной археологической литературе, так и в японской - что представляется слишком обобщенным и не отражающим разнообразия и функииональных особенностей материала. В качестве примера типологического подразделения кладов автор обращается к опыту спещиалистов, работающих с материалами докерамического неолита A (PPNA, 12-8,5 кал. тысс. л.н.) на Ближнем Востоке и в Передней Азии, а также с ритуальными комплексами бифасиальных изделий классического (III-X вв. н.э.) и постклассического (X-XVI вв. н.э.) периодов Месоамерики. Рассматриваются варианты разделения кладов на функииональные (депозиты, резервы), временно исключенные из технологической цепочки, и символические (вотивные, посвятительные, впускные), предназначенные исключительно для ритуальной сферы; намечаются перспективы дальнейших исследований в рамках данной тематики.

Ключевые слова: Дальний Восток, палеолит, неолит, клады каменных изделий, терминология, назначение, археологический контекст.

\author{
A.V. Tabarev \\ Institute of Archaeology and Ethnography SB RAS \\ Novosibirsk, Russia \\ E-mail:olmec@yandex.ru
}

\title{
Lithic Caches in the Paleolithic - Neolithic in the Far East: Terminological and Functional Aspects of Discussion
}

This article addresses the caches of stone artifacts of the Final Paleolithic - Neolithic/Jomon (35,000-2500 cal. BP), which have been found in the Russian part of the Far East and the Japanese Archipelago, focusing on terminology and functional purpose of the artifacts. There are less than dozen of such complexes in the Russian part (Novopetrovka culture on the Middle Amur, Osipovka culture on the Lower Amur, Ustinovska complexes in Primorie, and Ushki sites in Kamchatka), whereas there are over four hundred sites of the Final Paleolithic - Jomon on the Japanese Archipelago. Although caches significantly differ in terms of number of artifacts and their types, production techniques, location, and raw materials, in almost all cases, a single term is used for this category of finds both in Russian and Japanese archaeological literature. This term seems to be too general and does not reflect the diversity and specific functional features of the evidence. As an example of the typological division of caches, the author mentions the experience of specialists working with the evidence of Pre-Pottery Neolithic A (PPNA, 12,000-8500 cal. BP) in the Near East and Western Asia, as well as ritual complexes of bifacial artifacts belonging to the Classical (3rd-10th centuries AD) and Post-Classical (10th-15th centuries AD) periods in Mesoamerica. The variants proposed for dividing caches into functional (deposits, reserves), those temporarily excluded from the technological chain, and symbolic (votive, dedicatory, joint), intended exclusively for the ritual realm, are discussed, and prospects for further research of this topic are suggested.

Keywords: Far East, Paleolithic, Neolithic, lithic caches, terminology, meaning, archaeological context. 
По мнению большинства специалистов, для эпохи камня именно «клады» орудий и заготовок для различных изделий являются одной из наиболее интересных, информативных и, одновременно, непредсказуемых категорий находок. По сравнению с кладами последующих эпох палеометалла и средневековья, для которых постепенно нарабатываются критерии и закономерности расположения, локализация кладов палеолита - неолита и их функциональная нагрузка по-прежнему являются предметом дискуссий [Bradley, 2017].

В то время как наиболее яркие проявления практики создания кладов с исключительно сложной техникой изготовления артефактов (в первую очередь ретушированных наконечников) известны для эпохи солютре (22-17 кал. тыс. л.н.) в Западной Европе (клад Вольгю) и раннего палеоиндейского периода в Северной Америке (клады культуры кловис, 13-11 кал. тыс. л.н.) [Табарев, 2011; Clovis Caches..., 2014], в целом география распространения аналогичных находок существенно шире.

В данной работе мы обращаемся к кладам каменных изделий, известных на территории Дальнего Востока (российская часть, Японский архипелаг) для финала палеолита - неолита/дзёмона в диапазоне 35-2,5 кал. тыс. л.н. и к таким важным аспектам сопровождающей их дискуссии как терминология и функциональное назначение.

Это не первая публикация автора по тематике кладов в данном регионе - ранее нами рассматривались их специфические технологические характеристики [Kornfeld, Tabarev, 2009], возможность интерпретации кладов в качестве элемента погребального инвентаря [Табарев, Иванова, 2019], отдельные комплексы на Японском архипелаге [Kanomata, Tabarev, 2020], а также общее место кладов в технологии целых периодов, например, изначального дзёмона [Гладышев, Табарев, Цуцуми, 2021] и т. д. Истоки традиции дальневосточных кладов прослеживаются в позднем палеолите Севеpo-Восточной и Центральной Азии [Masojc et al., 2019; Tabarev et al., 2013].

Весьма показательна статистика по количеству кладов, зафиксированных на континентальной части российского Дальнего Востока и на Японских островах, которая, прежде всего, отражает степень изученности данного феномена. Для отечественной территории их насчитывается не более десятка (новопетровская культура на Среднем Амуре, осиповская на Нижнем Амуре, устиновские комплексы в Приморье и ушковские на Камчатке) [Деревянко, 1970; Диков, 1993; Dyakov, 1997; Tabarev et al., 2013], тогда как для финального палеолита - дзёмона на Японском архипелаге - более 400. Для 316 из них существуют детальная документация (отчеты о раскопках, фото, прорисовки) и хронологическая привязка, поэтому мы предпочитаем использовать именно эту цифру.

Итак, на островах Японского архипелага для финального палеолита (35-16 кал. тыс. л.н.) известно 13 кладов, для изначального дзёмона (16-10 кал. тыс. л.н.) - 23, для начального (10-7 кал. тыс. л.н.) - 24, для раннего (7-5,4 кал. тыс. л.н.) 33 , для среднего (5,4-4,3 кал. тыс. л.н.) - 137, для позднего (4,3-3,2 кал. тыс. л.н.) - 66, и для финального (3,2-2,4 кал. тыс. л.н.) - 20. Важно подчеркнуть, что клады встречаются по всей территории архипелага - от Хоккайдо до Кюсю [Kaner, Taniguchi, 2017; Kanomata, Tabarev, 2020].

Клады существенно различаются по количеству артефактов, их типу, локализации, сырью и т. д. Это видно даже по менее представительной подборке по комплексам на российской территории - клады нуклеусов в жилищах новопетровской культуры и на стоянке Ушки I, клад рубящих изделий на стоянке осиповской культуры Осиновая речка-10 в Приамурье и клад отщепов на стоянке Устиновка III в Приморье и т.д. Тем не менее, для обозначения этой категории находок используется практически один термин - как в отечественной археологической литературе (клад - зарытые, спрятанные ценности или нечто очень ценное для владельца и зарытое в землю), так и в японской (Intoku ikou или Heitan ikou - как эквивалент английскому термину cache - «тайник»). И русский, и японский термины представляются на данный момент слишком обобщенными и не отражающими разнообразия и функциональных особенностей материала.

В качестве примера типологического подразделения кладов можно обратиться, например, к опыту специалистов, работающих с материалами докерамического неолита А (PPNA, 12-8,5 кал. тыс. л.н.) на Ближнем Востоке и в Передней Азии. Перечень используемых терминов здесь гораздо шире, и все они являются элементами общей «технологической цепочки» (chaîne opératoire) - так, например, для кладов с однородным составом (заготовки нуклеусов, пластины, отщепы) применяется понятие «депозит» (франц. - dépôt, англ. - deposit), а для кладов, состоящих из различных видов заготовок, нуклеусов и готовых изделий - понятие «резерв» (франц. - réserve, англ. - reserve). При этом предполагается, что и «депозиты», и «резервы» имеют исключительно утилитарную нагрузку, лишь временно исключены из технологической цепочки и могут быть в зависимости от обстоятельств в нее в нужный момент возвращены. В противоположность этому, клады (caches) отражают исключительно символическую (ритуальную) сферу жизни и не являются возвратным элементом технологи- 
ческой цепочки. При их оценке первоочередное значение имеет не типологический состав предметов, а их размер, особое сырье и расположение (локализация) комплекса [Astruc, Ibáñez, GonzalezUrquijo, 2003].

Последний аспект детально разработан, например, в археологии классического (III-X вв. н.э.) и постклассического (X-XVI вв. н.э.) периодов Месоамерики применительно к кладам бифасиально обработанных изделий из обсидиана или высококачественного кремня, найденных при раскопках ритуальных сооружений. Эти клады подразделяют на «посвятительные» (англ. - dedicatory, votive caches), созданные до или в период строительства, и на «впускные» (англ. - intrusive caches), которые, выполняя иную ритуальную роль, периодически создавались в уже готовом сооружении [Kunen, Galindo, Chase, 2002].

Есть схемы, использующие все вышеназванные термины - в таких моделях все комплексы делятся на «клады» (caches) с символической нагрузкой (посвятительные, впускные, погребальные и т.д.) и на клады с функциональной нагрузкой (депозиты, резервы), для которых предлагается термин stock (русские эквиваленты-запас, склад) [Barzilai, Nigel Goring-Morris, 2007].

Нам представляется, что отдельные принципы этих схем могут быть использованы при типологическом подразделении кладов каменных изделий в дальневосточном регионе - во всяком случае, для кладов с зафиксированным положением в рамках памятника (в пределах жилища, в межжилищном пространстве, в погребении и т.д.). Гораздо сложнее с кладами, обнаруженными вне стоянок или мастерских - изменившийся ландшафт не позволяет определить изначальную привязку к маркеру (скале, ручью, гроту, отдельному дереву и т.д.) - их «биография» потребует анализа сырья, технологии, следов использования и других параметров всех составляющих клада, что, например, было продемонстрировано в публикации о недавно обнаруженном кладе бифасов начального дзёмона Касивабара на северо-востоке Японии [Kanomata, Tabarev, 2020] (см.рисунок).

Безусловно, для дальнейших исследований необходимо расширение данных о кладах финала палеолита - неолита в континентальной части Дальнего Востока, в первую очередь, за счет тер-
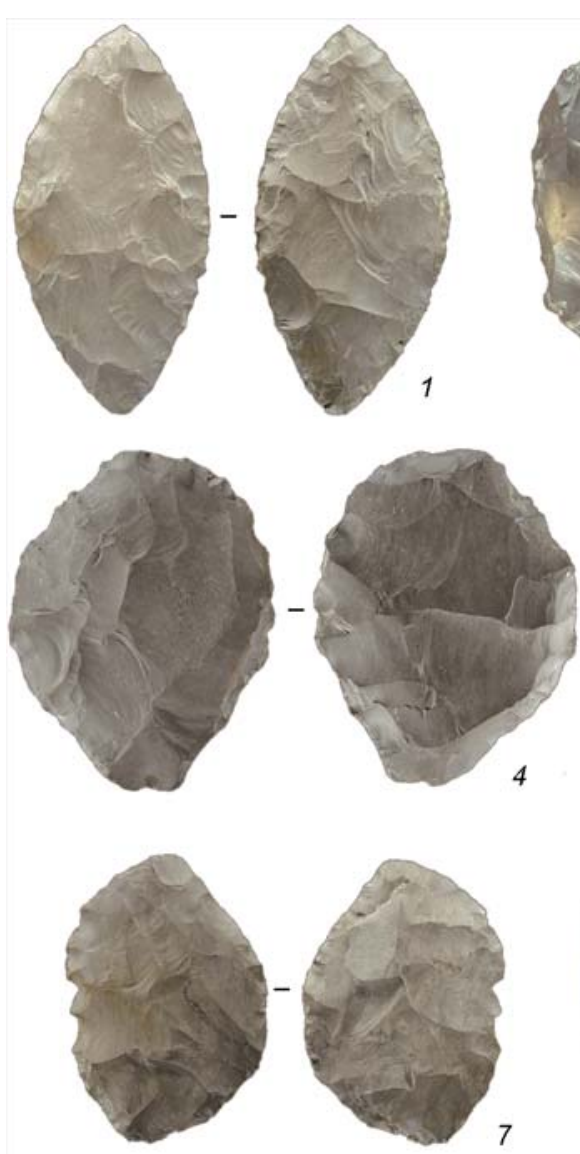
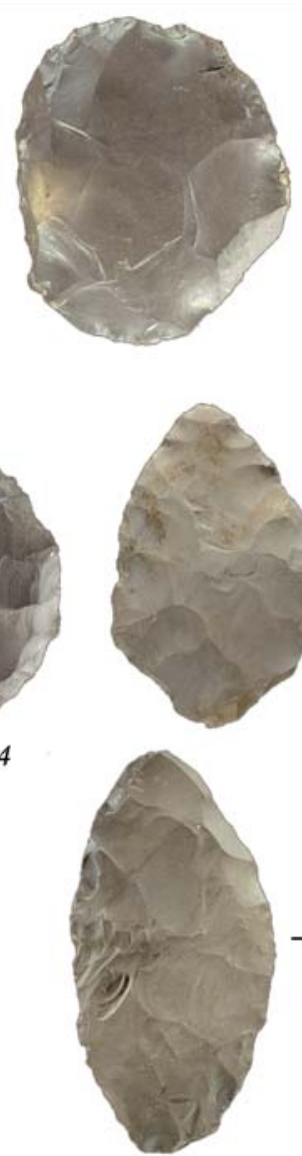

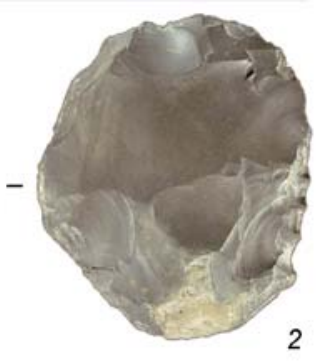

2
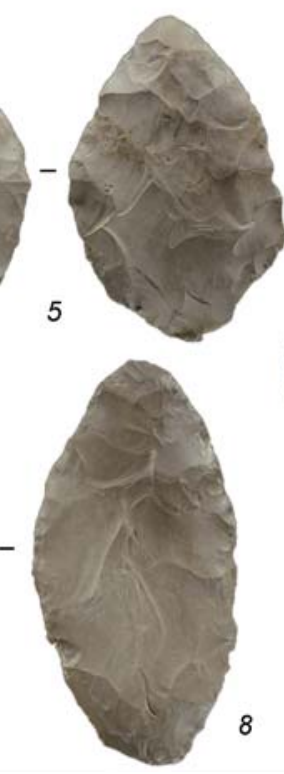
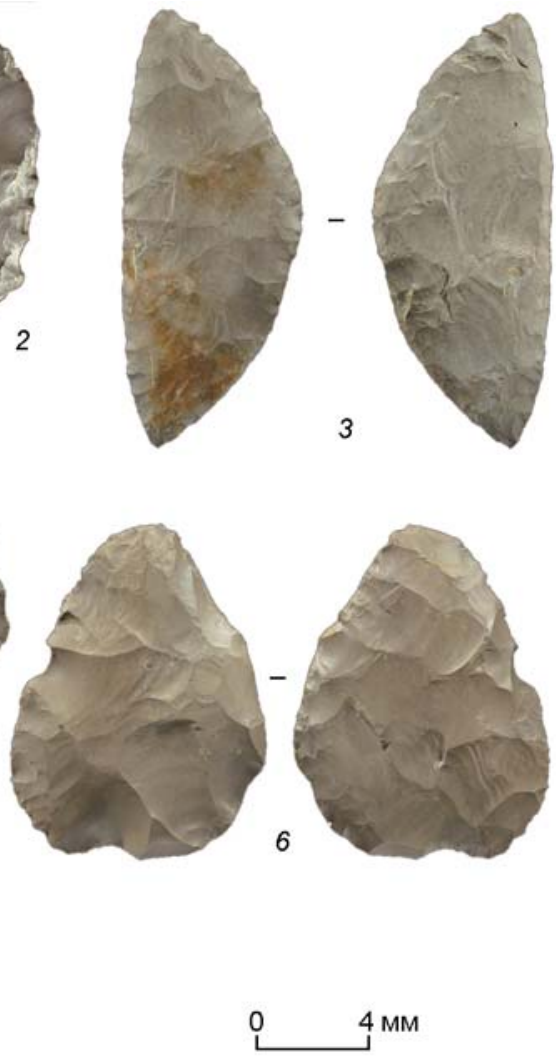

Клад бифасиально обработанных изделий Касивабара (1-8), изначальный дзёмон, префектура Ямагата, Япония [Kanomata, Taarev, 2020]. 
риторий Корейского полуострова и северо-востока Китая, по которым на сегодняшний день имеются лишь обрывочные сведения [Seong, 2015]. Не менее интересной представляется и перспектива детального сравнения кладов каменных изделий не только на сопредельных, но и географически удаленных территориях - недавний опыт такого исследования по североамериканским и западноевропейским комплексам подтверждает его продуктивность [Kilby, 2018].

\section{Благодарности}

Автор выражает благодарность своим японским коллегам проф. Й. Каномата (Университет Тохоку) и Т. Цуцуми (Музей Дзёмона Асама) за помощь с информацией и ценные комментарии по различным сюжетам подготовленной публикации.

\section{Список литературы}

Гладышев С.А., Табарев А.В., Цуцуми Т. Мир изначального дзёмона, Японский архипелаг: природа, технологии, общество, континентальные соседи // Stratum plus. - 2021. - №.1. - C. 337-358.

Деревянко А.П. Новопетровская культура Среднего Амура. - Новосибирск: Наука, 1970. - 204 с.

Диков Н.Н. Азия на стыке с Америкой в древности. СПб.: Наука, 1993. - 304 с.

Табарев А.В. Атлантическая сага, или Последнее путешествие солютрейцев (некоторые аспекты дискуссии о маршрутах первоначального заселения Нового Света) // Российский археологический ежегодник. - 2011. № 1. - С. 7-55.

Табарев А.В., Иванова Д.А. Наиболее ранние погребения на юге Дальнего Востока России и Японском архипелаге: вопросы идентификации и интерпретации // Проблемы археологии, этнографии, антропологии Сибири и сопредельных территорий: Материалы итоговой сессии Института археологии и этнографии СО РАН 2019 г. - Новосибирск: Изд-во ИАЭТ СО РАН, 2019. C .251-258.

Astruc L., Ibáñez J.J., Gonzalez-Urquijo J. "Dépôts", "réserves" et "caches" de matériel lithique taillé au Néolithique précéramique au Proche-Orient: Quelle gestion de l'outillage? // Paléorient. - 2003. - N. 1. - P. 59-78.

Barzilai O., Nigel Goring-Morris A. Bidirectional Blade and Tool Caches and Stocks in the PPNB of the Southern Levant // Systèmes techniques et communautés du Néolithique précéramique au Proche-Orient. - Actes du 5e colloque international, Fréjus, 2004. - Antibes: Éditions APDCA, 2007. - P. 277-294.

Bradley R. A Geography of Offerings: Deposits of valuables in the landscapes of ancient Europe. - Oxford: Oxbow Books, 2017. - 160 p.
Clovis Caches: Recent Discoveries and New Research.-UniversityofNewMexicoPress,Albuquerque,2014.$254 \mathrm{p}$

Dyakov V. The Oldest Habitation Site on the West Coast of the Sea of Japan // Current Research in the Pleistocene. 1997. - V. 14. - P. 20-21.

Kaner S., Taniguchi Y. The Development of Pottery and Associated Technological Developments in Japan, Korea, and the Russian Far East // Handbook of East and Southeast Asian Archaeology. - New York: Springer, 2017. P. 321-345.

Kanomata Y., Tabarev A. A newly discovered cache of large biface lithics from northern Honshu, Japan // Antiquity. - 2020. - Vol. 94 (374): e8, 1-8.

Kilby J. D. A North American Perspective on the Volgu Biface Cache from Upper Paleolithic France and its relationship to the "Solutrean Hypothesis" for Clovis Origins // Quaternary International. - 2018. V. 515. -P. 197-207.

Kornfeld M., Tabarev A. The French Connection? Or is It? // Current Research in the Pleistocene. - 2009. V. 26. -P. 90-92.

Kunen J. L., Galindo M. J., Chase E. Pits and bones: Identifying Maya ritual behavior in the archaeological record// Ancient Mesoamerica. -2002. - V. 13. - P. 197-211.

Masojc M., Szykulski J., Gunchinsuren B., Odsuren D., Winiarska-Kabacinska M., Szmit M. A Levalloisian jasper cache from the Arts Bogdyn Nuruu massif in the Gobi Altai Mountains, southern Mongolia // Comptes Rendus Palevol. - 2019. - V. 18. - P. 479-491.

Seong C. Diversity of Lithic Assemblages and Evolution of Late Palaeolithic Culture in Korea // Asian Perspectives. 2015. - V. 54. - P. 91-112.

Tabarev A.V., Gillam J.C., Kanomata Y., Gunchisuren B. A Paleolithic Cache at Tolbor (Northern Mongolia) // Archaeology, Ethnology and Anthropology of Eurasia. - 2013. - N. 3. - P. 14-21.

\section{References}

Astruc L., Ibáñez J.J., Gonzalez-Urquijo J. "Dépôts," "reserves" et "caches" de matériel lithique taillé au Néolithique précéramique au Proche-Orient: Quelle gestion de l'outillage? Paléorient, 2003, No. 1, pp. 59-78.

Barzilai O., Nigel Goring-Morris A. Bidirectional Blade and Tool Caches and Stocks in the PPNB of the Southern Levant. In Systèmes techniques et communautés du Néolithique précéramique au Proche-Orient. Actes du 5e colloque international, Fréjus, 2004. Antibes: Éditions APDCA, 2007, pp. 277-294.

Bradley R. A Geography of Offerings: Deposits of Valuables in the Landscapes of Ancient Europe. Oxford: Oxbow Books, 2017, 160 p.

Clovis Caches: Recent Discoveries and New Research. University of New Mexico Press, Albuquerque, 2014, 254 p. 
Derevianko A.P. Novopetrovskaya kultura Srednego Amura. Novosibirsk: Nauka, 1970, 204 p.

Dikov N.N. Aziya na styke s Amerikoi v drevnosti. St. Petersburg: Nauka, 1993, 304 p. (In Russ.).

Dyakov V. The Oldest Habitation Site on the West Coast of the Sea of Japan. Current Research in the Pleistocene, 1997, vol. 14, pp. 20-21.

Gladyshev S.A., Tabarev A.V., Tsutsumi T. Mir iznachalnogo dzemona, Yaponskiy arkhipelag: priroda, tekhnologii, obshchestvo, kontinentalnye sosedi. Stratum plus, 2021, No. 1, pp. 337-358. (In Russ.).

Kaner S., Taniguchi Y. The Development of Pottery and Associated Technological Developments in Japan, Korea, and the Russian Far East. In Handbook of East and Southeast Asian Archaeology, New York: Springer, 2017, pp. 321-345.

Kanomata Y., Tabarev A. A Newly Discovered Cache of Large Biface Lithics from Northern Honshu, Japan. Antiquity, 2020, vol. 94 (374), e8, pp. 1-8.

Kilby J. D. A North American Perspective on the Volgu Biface Cache from Upper Paleolithic France and its relationship to the "Solutrean Hypothesis" for Clovis Origins. Quaternary International, 2018, vol. 515, pp. 197-207.

Kornfeld M., Tabarev A. The French Connection? Or is It? Current Research in the Pleistocene, 2009, vol. 26, pp. 90-92.

Kunen J. L., Galindo M. J., Chase E. Pits and Bones: Identifying Maya Ritual Behavior in the Archaeological Record. Ancient Mesoamerica, 2002, vol. 13, pp. 197-211.
Masojc M., Szykulski J., Gunchinsuren B., Odsuren D., Winiarska-Kabacinska M., Szmit M. A Levalloisian Jasper Cache from the Arts Bogdyn Nuruu Massif in the Gobi Altai Mountains, Southern Mongolia. Comptes Rendus Palevol, 2019, vol. 18, pp. 479-491.

Seong C. Diversity of Lithic Assemblages and Evolution of Late Palaeolithic Culture in Korea. Asian Perspectives, 2015, vol. 54, pp. 91-112.

Tabarev A.V. Atlanticheskaya saga, ili Poslednee puteshestvie solyutreitsev (nekotorye aspekty diskussii o marshrutakh pervonachalnogo zaseleniya Novogo Sveta). Rossiyskiy arheologicheskiy ezhegodnik, 2011, No. 1, pp. 7-55. (In Russ.).

Tabarev A.V., Gillam J.C., Kanomata Y., Gunchisuren B. A Paleolithic Cache at Tolbor (Northern Mongolia). Archaeology, Ethnology and Anthropology of Eurasia, 2013, No. 3, pp. 14-21.

Tabarev A.V., Ivanova D.A. The Earliest Burials in the Southern Part of the Russian Far East and in the Japanese Archipelago: Problems of Identification and Interpretation. In Problems of Archaeology, Ethnography and Anthropology of Siberia and Neighboring Territories. Novosibirsk: IAE SB RAS Publ., 2019, pp. 251-258. (In Russ.).

Табарев A.B. https://orcid.org/0000-0002-6249-8057 